\title{
BMJ Open Association between excessive premature atrial complexes and cryptogenic stroke: results of a case- control study
}

\author{
Jithin K Sajeev, ${ }^{\oplus 1,2}$ Anoop N Koshy, ${ }^{1}$ Helen Dewey, ${ }^{1,3}$ Jonathan M Kalman, ${ }^{4}$ \\ Kevin Rajakariar, ${ }^{2}$ Mae C Tan, ${ }^{2}$ Maryann Street, ${ }^{\circ, 6}$ Louise Roberts, ${ }^{1,2}$ \\ Jennifer C Cooke, ${ }^{1,2}$ Michael Wong, ${ }^{2}$ Tanya Frost, ${ }^{7}$ Andrew W Teh ${ }^{1,2}$
}

To cite: Sajeev JK, Koshy AN, Dewey $\mathrm{H}$, et al. Association between excessive premature atrial complexes and cryptogenic stroke: results of a case-control study. BMJ Open 2019;9:e029164. doi:10.1136/ bmjopen-2019-029164

- Prepublication history and additional material for this paper are available online. To view please visit the journal (http:// dx.doi.org/10.1136/bmjopen2019-029164).

Received 15 January 2019 Revised 14 March 2019 Accepted 25 June 2019

Check for updates

(C) Author(s) (or their employer(s)) 2019. Re-use permitted under CC BY-NC. No commercial re-use. See rights and permissions. Published by BMJ.

For numbered affiliations see end of article.

Correspondence to Dr Andrew W Teh;

Andrew.teh@easternhealth. org.au

\section{ABSTRACT}

Objective Recent anticoagulation trials in all-comer cryptogenic stroke patients have yielded equivocal results, reinvigorating the focus on identifying reproducible markers of an atrial myopathy. We investigated the role of excessive premature atrial complexes (PACs) in ischaemic stroke, including cryptogenic stroke and its association with vascular risk factors.

Methods and results A case-control study was conducted utilising a multicentre institutional stroke database to compare 461 patients with an ischaemic stroke or transient ischaemic attack (TIA) with a control group consisting of age matched patients without prior history of ischaemic stroke/TIA. All patients underwent 24-hour Holter monitoring during the study period and atrial fibrillation was excluded. An excessive PAC burden, defined as $\geq 200$ PACs/24 hours, was present in $25.6 \%$ and $14.7 \%(p<0.01)$, of stroke/TIA and control patients, respectively. On multivariate regression, excessive PACs (OR 1.97; 95\% Cl 1.29 to 3.02; $\mathrm{p}<0.01$ ), smoking (OR $1.58 ; 95 \% \mathrm{Cl} 1.06$ to $2.36 ; \mathrm{p}<0.05)$ and hypertension (OR 1.53; $95 \% \mathrm{Cl} 1.07$ to 2.17 ; $p<0.05$ ) were independently associated with ischaemic stroke/TIA. Excessive PACs remained the strongest independent risk factor for the cryptogenic stroke subtype (OR 1.95; 95\% Cl 1.16 to 3.28; $p<0.05)$. Vascular risk factors that promote atrial remodelling, increasing age ( $\geq 75$ years, OR $3.64 ; 95 \% \mathrm{Cl}$ 2.08 to $6.36 ; \mathrm{p}<0.01$ ) and hypertension (OR $1.54 ; 95 \% \mathrm{Cl}$ 1.01 to $2.34 ; p<0.05)$ were independently associated with excessive PACs.

Conclusions Excessive PACs are independently associated with cryptogenic stroke and may be a reproducible marker of atrial myopathy. Prospective studies assessing their utility in guiding stroke prevention strategies may be warranted.

\section{INTRODUCTION}

Approximately 100000 strokes occur every year in the UK, with 1 in 4 survivors experiencing another stroke. ${ }^{1}$ While $87 \%$ of all strokes are ischaemic in nature, $25 \%-35 \%$ of these are labelled cryptogenic, as a clear cause is not identified. ${ }^{2}{ }^{3}$ Subclinical paroxysmal
Strengths and limitations of this study

- This study employed a case-control design to compare the burden of premature atrial complexes (PACs) in ischaemic stroke, including the cryptogenic stroke subtype and an age-matched control group.

- All patients underwent 24 hours of ambulatory Holter monitoring to exclude atrial fibrillation and to document the burden of PACs.

- This study describes the association between vascular risk factors and PACs and used multivariate analysis to reduce confounding.

- The study is limited by its cross-sectional, casecontrol design and causality cannot be inferred from the associations.

atrial fibrillation (AF) is postulated to be the cause for a significant proportion of these cryptogenic strokes. ${ }^{4}{ }^{5}$ However, despite prolonged rhythm monitoring, occult AF occur in only a small proportion of patients. ${ }^{67}$

With the recent equivocal results of randomised controlled anticoagulation trials in all-comer patients with embolic stroke of undetermined source, there has been a heightened focus in identifying reproducible markers of an atrial myopathy. ${ }^{8}$ Premature atrial complexes (PACs) have been thought to be a benign phenomenon with a prevalence in the general population that ranges from $6 \%$ to $29 \% .{ }^{9}$ A limited number of studies have shown a significant association between excessive PACs and ischaemic stroke, suggesting their relevance as a marker of atrial myopathy. ${ }^{10-14}$ While another study has shown an elevated risk for recurrent stroke in patients with excessive PACs, following a cryptogenic stroke. ${ }^{15}$ However, these have not delineated whether baseline excessive PACs confer an increased risk for the cryptogenic 
stroke subtype. In addition, it is unclear whether vascular risk factors that promote stroke, independently and uniformly lead to atrial remodelling that result in excessive PAC burden. We sought to determine the association between excessive PACs and ischaemic stroke, including the cryptogenic stroke subtype and their relationship to conventional risk factors.

\section{METHODS}

A multicentre case-control study was conducted among consecutive patients who presented with an ischaemic stroke or transient ischaemic attack (TIA) between May 2011 and December 2015. Patients within the stroke/TIA group were identified through a prospectively maintained institutional stroke database that covered three tertiary, university hospitals. Stroke subtypes were determined according to the Trial of Org 10172 in Acute Stroke Treatment classification. ${ }^{16}$

Inclusion criteria were (1) age $\geq 18$ years; (2) adjudicated to have had an ischaemic stroke/TIA by the stroke service and (3) underwent 24-hour Holter monitoring following their index stroke/TIA. Exclusion criteria were (1) history of AF, atrial flutter or a subsequent diagnosis of these arrhythmias on inpatient telemetry, Holter monitoring or during follow-up; (2) underlying severe cardiomyopathy with an ejection fraction $<35 \%$; (3) previous coronary artery bypass grafting; (4) recent myocardial infarction or (5) severe chronic obstructive airways disease.

Eligible patients were compared with a group of age-matched controls, without prior history of stroke or $\mathrm{AF}$ and underwent outpatient Holter monitoring. The control group were composed of patients who underwent investigation of chest pain, syncope, presyncope and palpitations. The stroke/TIA and control groups were age matched with the same exclusion criteria applied.

\section{Clinical assessment and outcome measures}

All included patients adjudicated as having a stroke/ TIA underwent investigation and treatment as per the national stroke guidelines recommendation for standard of care. ${ }^{17}$ This included physical examination, blood measurements, 12 lead ECG, pulse oximetry, CT of the brain, inpatient cardiac monitoring and vascular assessment. All patients underwent Holter monitoring with 24 hours of continuous rhythm capture utilising the SEER Light Holter Monitor (GE Healthcare, Milwaukee, USA). Data were analysed offline on completion of the monitoring period by cardiac technicians and subsequently reviewed by a cardiac electrophysiologist blinded to the study hypothesis. Rhythm analysis was conducted using MARS Ambulatory ECG Analysis System (GE Healthcare).

Baseline demographic and clinical data were collected from electronic health records along with vascular risk factors to allow for calculation of a $\mathrm{CHA}_{2} \mathrm{DS}_{2}$ VASc score, medication use and to identify presentations with recurrent stroke or TIA. Based on prior literature, we defined excessive PAC burden as $\geq 200$ PACs/24hours and a long atrial run as $\geq 20$ beats. $^{101819}$

\section{Statistical methods}

Demographic data, disease status and outcome measures are presented as proportions and summarised by descriptive statistics. Data were tested for normality and parametric or non-parametric tests applied as appropriate. Correlation trends were analysed using Spearman's rho for non-parametric data. A $p$ value $<0.05$ was deemed statistically significant and a $95 \%$ CI is presented where applicable. Markers associated with stroke/TIA and excessive PACs were identified by univariate and multivariate logistical regression. Any variable with a $p$ value $<0.25$ on univariate analysis was included in multivariate analyses. All statistical analysis was performed with SPSS Statistics V.24.0.

\section{Patient and public involvement}

Public involvement was sought after the methods and outcome measures were identified. The protocol and study design were reviewed by human research ethics committee, $45 \%$ of whom were members of the public.

\section{Ethics approval and data sharing}

The raw data will be made available by the corresponding author on reasonable request.

\section{RESULTS}

In total, 537 patients presented with a stroke/TIA during the study inclusion period and underwent Holter monitoring. Twenty-three out of 537 (4.2\%) patients had AF identified on Holter monitoring and were excluded. Four patients with AF had an excessive PAC burden (17\%).

Following exclusions, 461 patients with a stroke/TIA were compared against 251 age-matched patients who underwent Holter monitoring during the same time period (figure 1).

The median time to Holter monitoring following the stroke or TIA was 40 days.

\section{Ischaemic stroke and PAC burden}

Baseline characteristics stratified according to study groups are shown in table 1 . In both groups, the mean age was 70 years and the majority of patients were male. Stroke/TIA patients were significantly more likely to have comorbidities of hypertension, diabetes mellitus, dyslipidaemia, peripheral vascular disease and a prior history of smoking. There were 79 patients with a prior cerebrovascular event in the stroke/TIA group. On admission, there was significantly higher use of statins in the stroke/ TIA cohort; however, no difference was evident in the use of antiplatelet therapy or oral anticoagulants. The prevalence of excessive PACs were significantly higher in the stroke/TIA group (25.6\% vs $14.7 \%, \mathrm{p}=0.001$ ); however, atrial runs of $\geq 20$ beats were not significantly different (table 1). 


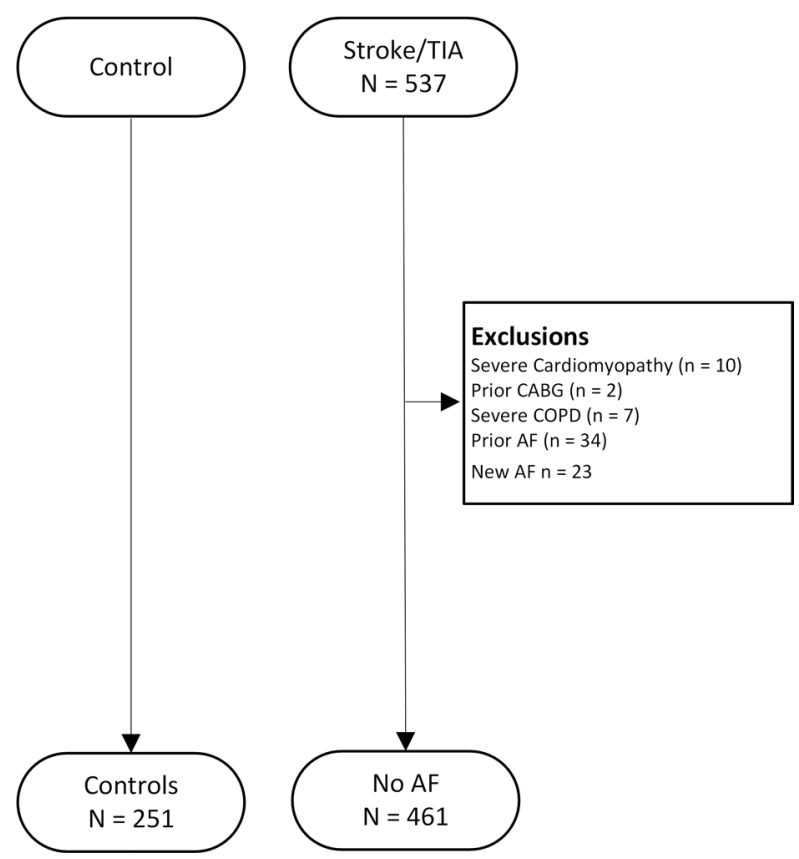

Figure 1 Patient selection: inclusions and exclusions. AF, atrial fibrillation; CABG, coronary artery bypass grafting; COPD, chronic obstructive pulmonary disease; TIA, transient ischaemic attack.

Multivariate analysis showed female sex, hypertension, history of smoking and excessive PACs were significantly associated with a stroke/TIA. Excessive PACs conferred the highest risk for stroke/TIA with an OR of 1.97 (95\% CI 1.29 to 3.02), but the difference was not significant when compared with other risk factors associated with stroke/TIA (table 2). Multivariate analysis with various definitions of excessive PACs based on prior literature yielded similar results, with a significant association between excessive PACs and stroke/TIA (online supplementary file 1).

Analysis of PAC burden revealed a skewed distribution, median PACs/24hours and longest atrial ectopic runs were significantly higher in the stroke/TIA group. However, number of beats in runs of $>3$ beats was not significantly different between the two groups.

\section{Cryptogenic stroke and PAC burden}

One hundred and eighty-five patients with cryptogenic stroke, after excluding TIA, were compared with 251 patients in the control group. The mean age was not significantly different between the control and cryptogenic stroke group, 70.5 and 68.9 years, respectively. Cryptogenic stroke was significantly associated with excessive PAC burden, female sex, hypertension, diabetes mellitus, smoking, obstructive sleep apnoea and a higher median $\mathrm{CHA}_{2} \mathrm{DS}_{2}$ VASc score on univariate analysis. Excessive PACs (OR: 1.95 ; $95 \%$ CI 1.16 to 3.28 ), female sex (OR: 1.78; $95 \%$ CI 1.19 to 2.67 ) and hypertension (OR: 1.67; $95 \%$ CI 1.05 to 2.64 ) maintained significant and independent associations with cryptogenic stroke subtype on multivariate logistical regression (table 2).
Vascular risk factors and PAC burden

Increasing $\mathrm{CHA}_{2} \mathrm{DS}_{2} \mathrm{VASc}$ score was associated with increasing median PACs in both groups (figure 2). A $\mathrm{CHA}_{2} \mathrm{DS}_{2}$ VASc score $>3$ in the control group and two in the stroke/TIA group were associated with an excessive PAC burden. However, only a moderate to weak correlation was evident between increasing $\mathrm{CHA}_{2} \mathrm{DS}_{2}$ VASc score and increasing PACs/24hours in all patients $\left(r_{\mathrm{s}}=0.32\right.$, $\mathrm{p}<0.001)$, control patients $\left(\mathrm{r}_{\mathrm{s}}=0.24, \mathrm{p}<0.001\right)$ and stroke/ TIA patients $\left(r_{\mathrm{s}}=0.32, \mathrm{p}<0.001\right)$, respectively.

In all patients, age, hypertension, diabetes mellitus and peripheral vascular disease were significant univariate predictors of excessive PACs. However, only age and hypertension remained independently associated with excessive PACs on regression analysis, with age $\geq 75$ years being the strongest marker associated with excessive PACs (table 3).

\section{DISCUSSION}

In this study, we compared the differences in PAC burden between patients with a stroke/TIA and an age-matched control population, after excluding AF. Excessive PAC burden was significantly more common in the stroke/ TIA group. An important new finding in our study was that excessive PACs demonstrated an independent association for the cryptogenic stroke subtype, after adjusting for conventional risk factors (OR: 1.95; 95\% CI 1.16 to 3.28). There was a stepwise rise in PAC burden with increasing number of vascular risk factors; age and hypertension were independent risk factors associated with excessive PACs.

Investigators have previously demonstrated a significantly higher PAC burden in patients who develop incident $\mathrm{AF}$ and ischaemic stroke. ${ }^{18}{ }^{20}$ Existing longitudinal studies from Engström et al that demonstrated a high PAC burden conferred a 1.9 times higher risk for ischaemic stroke. ${ }^{19}$ Despite the differences in methodology, the current study showed 1.97 times rise in odds for ischaemic stroke. Prior studies have also shown an association between runs of PACs and ischaemic stroke in patients without documented $\mathrm{AF}^{21}$ The Copenhagen Holter study, a cohort study that analysed the risk for stroke with an elevated PAC burden, defined excessive supraventricular ectopic activity as a composite of either $>30$ PACs/hour or a run of $>20$ PACs, and found a positive correlation with increased stroke and death. ${ }^{10} 13$ In contrast, we did not show a significant difference in PAC runs $>20$ beats between the two groups. ${ }^{15}$ This apparent discrepancy is likely due to a lack of standardised definitions for excessive PACs and treating atrial premature runs $\geq 20$ beats as a standalone variable in the current study, instead of a composite measure.

The present study specifically analysed the association between excessive PACs and cryptogenic stroke subtype. It demonstrated an independent association between cryptogenic stroke subtype and excessive PACs with an OR of 1.95. This is an important finding and lends further 
Table 1 Baseline characteristics of study participants

\begin{tabular}{|c|c|c|c|}
\hline Characteristics & $\begin{array}{l}\text { Control } \\
n=251 \\
n(\%)\end{array}$ & $\begin{array}{l}\text { Stroke/TIA } \\
\mathrm{n}=461 \\
\mathrm{n}(\%)\end{array}$ & $P$ value \\
\hline Age, years (SD) & $70.5(11.6)$ & $69.8(12.5)$ & 0.45 \\
\hline Sex, female & $88(35.1)$ & $195(42.3)$ & 0.06 \\
\hline \multicolumn{4}{|l|}{ Stroke subtypes } \\
\hline Large vessel atherosclerosis & - & $82(17.8)$ & - \\
\hline Small vessel occlusion & - & $86(18.7)$ & - \\
\hline Cryptogenic & - & $291(63.1)$ & - \\
\hline Stroke of other determined aetiology & - & $2(0.4)$ & - \\
\hline \multicolumn{4}{|l|}{ Vascular risk factors } \\
\hline Hypertension & $130(51.8)$ & $294(63.8)$ & 0.01 \\
\hline Dyslipidaemia & $91(36.3)$ & $209(45.3)$ & 0.02 \\
\hline Diabetes mellitus & $38(15.1)$ & $113(24.5)$ & 0.01 \\
\hline Any smoking & $44(17.5)$ & $118(25.6)$ & 0.01 \\
\hline Previous stroke/TIA & $0(0)$ & $79(17.1)$ & $<0.001$ \\
\hline Myocardial infarction & $40(15.9)$ & $82(17.8)$ & 0.53 \\
\hline Peripheral vascular disease & $5(2.0)$ & $26(5.6)$ & 0.02 \\
\hline Sleep apnoea & $14(5.6)$ & $13(2.8)$ & 0.07 \\
\hline History of heart failure & $11(4.4)$ & $25(5.4)$ & 0.55 \\
\hline $\mathrm{CHA}_{2} \mathrm{DS}_{2}$ VASc score, median (IQR) & $2(1-3)$ & $5(4-5)$ & $<0.001$ \\
\hline \multicolumn{4}{|l|}{ Medications } \\
\hline Warfarin & $4(1.6)$ & $4(0.9)$ & 0.38 \\
\hline Direct oral anticoagulant & $4(1.6)$ & $1(0.2)$ & - \\
\hline Antiplatelet therapy & $81(32.3)$ & $165(35.8)$ & 0.35 \\
\hline Beta blocker & $48(19.1)$ & $82(17.8)$ & 0.66 \\
\hline Ace inhibitor & $105(41.8)$ & $224(48.6)$ & 0.08 \\
\hline Statin & $79(31.5)$ & $187(40.6)$ & 0.02 \\
\hline \multicolumn{4}{|l|}{ Premature atrial complexes } \\
\hline PACs/24 hours, median (IQR) & $37(13-115)$ & $62(20-208)$ & $<0.01$ \\
\hline Longest atrial run, median (IQR) & $3(0-7)$ & $3(0-8)$ & $<0.01$ \\
\hline Atrial runs $>3$ beats, median (IQR) & $1(0-2)$ & $1(0-4)$ & 0.07 \\
\hline$\geq 200$ PACs/24hours & $37(14.7)$ & $118(25.6)$ & $<0.001$ \\
\hline$\geq 20$ beats in runs & $13(5.2)$ & $27(5.9)$ & 0.71 \\
\hline
\end{tabular}

The data are presented as $\mathrm{n}(\%)$, unless otherwise stated.

PACs, premature atrial complexes; TIA, transient ischaemic attack.

support to the hypothesis, that excessive PACs may be the manifestation or marker of underlying atrial myopathy, that confers an increased risk for cryptogenic stroke. Further, similar to AF, the risk for ischaemic stroke in the presence of an excessive PAC burden appear to be modulated by vascular risk factors. $\mathrm{A} \mathrm{CHA}_{2} \mathrm{DS}_{2}$ VASc score of 2 conferred a similar risk for ischaemic stroke in patients with excessive PACs, as with AF. ${ }^{10}$

An increasing $\mathrm{CHA}_{2} \mathrm{DS}_{2}$ VASc score was significantly associated with increasing median PAC burden in both the control and stroke/TIA group. However, despite the positive correlation between $\mathrm{CHA}_{2} \mathrm{DS}_{2} \mathrm{VASc}$ score and
PACs, the strength of the correlation itself remained weak. This was suggestive of differential effects of the various components of $\mathrm{CHA}_{2} \mathrm{DS}_{2} \mathrm{VASc}$ score in contributing to a high PAC burden. The independent contribution of the various risk markers that make up $\mathrm{CHA}_{2} \mathrm{DS}_{2}$ VASc score have not been assessed previously. ${ }^{13}{ }^{12}$ Delineation of these specific risk factors that contribute to excessive PACs provides insights into the potential pathophysiological basis for excessive PAC.

Increasing age and hypertension were independently and significantly associated with excessive PACs in the present study. This is consistent with electroanatomical 
Table 2 Multivariate analysis: risk factors associated with stroke/TIA and cryptogenic stroke

\begin{tabular}{|c|c|c|c|c|}
\hline \multirow[b]{2}{*}{ Characteristic } & \multicolumn{2}{|l|}{ Stroke/TIA } & \multicolumn{2}{|l|}{ Cryptogenic stroke } \\
\hline & OR $(95 \% \mathrm{Cl})$ & $P$ value & OR $(95 \% \mathrm{Cl})$ & $P$ value \\
\hline Female & 1.51 (1.09 to 2.11$)$ & $<0.05$ & 1.78 (1.19 to 2.67$)$ & $<0.01$ \\
\hline Smoking & 1.58 (1.06 to 2.36$)$ & $<0.05$ & 1.55 (0.95 to 2.53$)$ & 0.08 \\
\hline$\geq 200$ PACs & 1.97 (1.29 to 3.02$)$ & $<0.01$ & 1.95 (1.16 to 3.28$)$ & $<0.05$ \\
\hline
\end{tabular}

Variables adjusted in the multivariate model: age, sex, excessive premature atrial complexes, hypertension, diabetes mellitus, smoking, dyslipidaemia, peripheral vascular disease and sleep apnoea.

PACs, premature atrial complexes; TIA, transient ischaemic attack.

studies that demonstrated slower conduction velocities and both global and regional reduction in atrial voltages with increasing age and hypertension. ${ }^{23}{ }^{24}$ Such areas corresponded to delayed enhancement on MRI and histological fibrosis. ${ }^{25}{ }^{26}$ Thrombogenesis associated with this underlying atrial remodelling may help explain a significant proportion of strokes currently classified as cryptogenic. Both advancing age and hypertension are also associated with small and large vessel stroke subtypes. In addition to atrial remodelling, it is likely that vascular risk factors promote thrombogenesis and ischaemic stroke through multiple pathways including arterial endothelial dysfunction, and atherosclerosis with localised plaque rupture. ${ }^{27}$

Our report of the independent association between excessive PACs and cryptogenic stroke further implicates a risk factor driven atrial substrate abnormality in its pathogenesis. These findings are clinically relevant as the results of a recently concluded large multicentre randomised controlled trial failed to demonstrate a benefit for oral anticoagulation in an unselected population with embolic stroke of undetermined source. ${ }^{8}$ This highlights the heterogeneity of the pathophysiological mechanisms that lead to cryptogenic stroke. There is an unmet clinical need to develop risk markers that identify the subset of patients with cryptogenic stroke that occur as a result of cardioembolism.

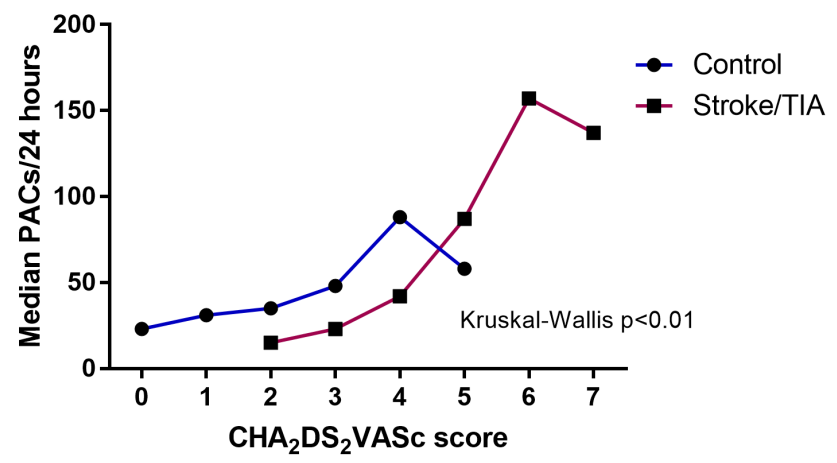

Figure 2 Median PACs by $\mathrm{CHA}_{2} \mathrm{DS}_{2}$ VASc score for control and stroke/TIA groups. CHA $\mathrm{DS}_{2}$ VASc, riskscore for ischaemic stroke; PACs, premature atrial complexes; TIA, transient ischaemic attack.
Studies have previously described serological and echocardiographic markers associated with the recurrence of cryptogenic stroke. ${ }^{28}$ Similarly, excessive PACs are readily assessed and may serve as a novel and reproducible marker to identify patients at high risk for the cryptogenic stroke. It is unclear if excessive PACs directly promote thrombogenesis or if they are simply a marker of adverse atrial remodelling that leads to thrombogenesis and stroke. Regardless, the risk conferred by an elevated $\mathrm{CHA}_{2} \mathrm{DS}_{2}$ VASc score in conjunction with excessive PACs for ischaemic stroke remains significant. ${ }^{10}$

This study has limitations. The absence of prolonged monitoring with devices such as implantable loop recorders could have led to an underestimation of incident AF. However, we excluded all patients with a diagnosis of AF over 1.9 years of mean follow-up. A higher number of cryptogenic stroke patients were present in our population than previously reported. This is likely due to referral bias, as patients were only included if they underwent Holter monitoring. All patients included in the study had guideline-based referral for Holter monitoring. However, as Holter monitoring was an inclusion criterion, we do not have data on patient who may have received their Holter monitoring at an external institution. However, the higher prevalence of cryptogenic stroke improves the strength of our findings in this specific subset of stroke patients. The time to Holter monitoring following the stroke, based on routine institutional clinical waiting periods, could have introduced unintended

\begin{tabular}{|c|c|c|}
\hline Risk factor & OR $(95 \% \mathrm{Cl})$ & $P$ value \\
\hline \multicolumn{3}{|l|}{ Age (years) } \\
\hline 65-74 & 2.52 (1.42 to 4.45$)$ & $<0.01$ \\
\hline$\geq 75$ & 3.64 (2.08 to 6.36 ) & $<0.01$ \\
\hline Hypertension & 1.54 (1.01 to 2.34$)$ & $<0.05$ \\
\hline Diabetes mellitus & 1.41 (0.91 to 2.20$)$ & 0.13 \\
\hline
\end{tabular}

Variables adjusted in the multivariate model: age: $<65,65-$ $74, \geq 75$ years, gender, hypertension, diabetes mellitus, smoking, dyslipidaemia, peripheral vascular disease, sleep apnoea. PACs, premature atrial complexes. 
variables such as neurologically mediated cardiac modelling with resultant excessive PACs and reverse causality. The higher burden of PACs was noted in a highly selective patient cohort with ischaemic stroke and a high burden of vascular risk factors. Despite the use of multivariate regression analysis, unrecognised confounders cannot be excluded in a cross-sectional case-control study, therefore these findings should not be extrapolated to other patient cohorts.

\section{CONCLUSIONS}

Excessive PACs are significantly associated with cryptogenic stroke. Vascular risk factors, increasing age and hypertension, were independently associated with excessive PACs. The utility of novel and reproducible cardiac markers to guide preventative strategies in cryptogenic stroke warrant further evaluation.

\section{Author affiliations}

${ }^{1}$ Eastern Health Clinical School, Monash University, Box Hill, Victoria, Australia

${ }^{2}$ Department of Cardiology, Eastern Health, Box Hill, Victoria, Australia

${ }^{3}$ Neurosciences, Eastern Health, Box Hill, Victoria, Australia

${ }^{4}$ Royal Melbourne Hospital, Melbourne, Victoria, Australia

${ }^{5}$ School of Nursing and Midwifery, Deakin University, Burwood, Victoria, Australia

${ }^{6}$ Deakin University Nursing and Midwifery Research Centre, Eastern Health, Box Hill, Victoria, Australia

${ }^{7}$ Department of Neurosciences, Eastern Health, Box Hill, Victoria, Australia

Contributors JKS led and designed the study, collated and analysed data and wrote the manuscript. ANK, KR, MCT and TF collated and analysed the data and revised the manuscript. HD, JMK, LR, JCC and MW contributed to study design and revised the manuscript. MS analysed the data and revised the manuscript. AWT supervised and designed the study revised the manuscript. All authors read and approved the final manuscript.

Funding The authors have not declared a specific grant for this research from any funding agency in the public, commercial or not-for-profit sectors.

\section{Competing interests None declared.}

Patient consent for publication Not required.

Ethics approval The research protocol was approved by the institutional Human Research Ethics Committee and written informed consent was not deemed necessary by the committee (approval number: LR09/2016).

Provenance and peer review Not commissioned; externally peer reviewed.

Open access This is an open access article distributed in accordance with the Creative Commons Attribution Non Commercial (CC BY-NC 4.0) license, which permits others to distribute, remix, adapt, build upon this work non-commercially, and license their derivative works on different terms, provided the original work is properly cited, appropriate credit is given, any changes made indicated, and the use is non-commercial. See: http://creativecommons.org/licenses/by-nc/4.0/.

\section{REFERENCES}

1. Royal College of Physicians Sentinel Stroke National Audit Programme (SSNAP). National clinical audit annual results portfolio March 2016-April 2017. 2017. http://bit.ly/1NHYlqH (accessed 24 Dec 2018)

2. Hart RG, Diener HC, Coutts SB, et al. Embolic strokes of undetermined source: the case for a new clinical construct. Lancet Neurol 2014;13:429-38.

3. Benjamin EJ, Blaha MJ, Chiuve SE, et al. Heart disease and stroke statistics-2017 update: a report from the American Heart Association. Circulation 2017;135:e146-e603.
4. Gladstone DJ, Spring M, Dorian P, et al. Atrial fibrillation in patients with cryptogenic stroke. N Engl J Med 2014;370:2467-77.

5. Ntaios G, Papavasileiou V, Milionis H, et al. Embolic strokes of undetermined source in the Athens stroke registry: a descriptive analysis. Stroke 2015;46:176-81.

6. Liao J, Khalid Z, Scallan C, et al. Noninvasive cardiac monitoring for detecting paroxysmal atrial fibrillation or flutter after acute ischemic stroke: a systematic review. Stroke 2007;38:2935-40.

7. Brachmann J, Morillo CA, Sanna T, et al. Uncovering atrial fibrillation beyond short-term monitoring in cryptogenic stroke patients: three-year results from the cryptogenic stroke and underlying atrial fibrillation trial. Circ Arrhythm Electrophysiol 2016;9:e003333.

8. Hart RG, Sharma M, Mundl H, et al. Rivaroxaban for stroke prevention after embolic stroke of undetermined source. $N$ Engl J Med 2018;378:2191-201.

9. Conen D, Adam M, Roche F, et al. Premature atrial contractions in the general population: frequency and risk factors. Circulation 2012;126:2302-8.

10. Larsen BS, Kumarathurai P, Falkenberg J, et al. Excessive atrial ectopy and short atrial runs increase the risk of stroke beyond incident atrial fibrillation. J Am Coll Cardiol 2015;66:232-41.

11. Marinheiro R, Parreira L, Amador P, et al. Excessive atrial ectopic activity as an independent risk factor for ischemic stroke. Int $J$ Cardiol 2017;249:226-30.

12. Himmelreich JCL, Lucassen WAM, Heugen M, et al. Frequent premature atrial contractions are associated with atrial fibrillation, brain ischaemia, and mortality: a systematic review and metaanalysis. Europace 2018. doi: 10.1093/europace/euy276.[Epub ahead of print 1 Dec 2018].

13. Binici Z, Intzilakis T, Nielsen OW, et al. Excessive supraventricular ectopic activity and increased risk of atrial fibrillation and stroke. Circulation 2010;121:1904-11.

14. Sejr MH, Riahi S, Larsen TB, et al. Premature atrial complexes in an ischemic stroke population and risk of recurrent stroke: a systematic review. Expert Rev Cardiovasc Ther 2017;15:447-55.

15. Pinho J, Braga CG, Rocha S, et al. Atrial ectopic activity in cryptogenic ischemic stroke and TIA: a risk factor for recurrence. $J$ Stroke Cerebrovasc Dis 2015;24:507-10.

16. Adams HP, Bendixen BH, Kappelle LJ, et al. Classification of subtype of acute ischemic stroke. Definitions for use in a multicenter clinical trial. TOAST. Trial of Org 10172 in Acute Stroke Treatment. Stroke 1993;24:35-41.

17. National Stroke Foundation Melbourne Australia. Clinical guidelines for stroke management 2010, 2010.

18. Todo K, Moriwaki H, Saito K, et al. Frequent premature atrial contractions in stroke of undetermined etiology. Eur Neurol 2009;61:285-8.

19. Engström G, Hedblad B, Juul-Möller S, et al. Cardiac arrhythmias and stroke: increased risk in men with high frequency of atrial ectopic beats. Stroke 2000;31:2925-9.

20. Acharya T, Tringali S, Bhullar M, et al. Frequent atrial premature complexes and their association with risk of atrial fibrillation. Am J Cardiol 2015;116:1852-7.

21. Murakoshi N, Xu D, Sairenchi T, et al. Prognostic impact of supraventricular premature complexes in community-based health checkups: the Ibaraki Prefectural Health Study. Eur Heart J 2015;36:170-8.

22. Kamel H, Elkind MS, Bhave PD, et al. Paroxysmal supraventricular tachycardia and the risk of ischemic stroke. Stroke 2013;44:1550-4.

23. Kistler PM, Sanders P, Fynn SP, et al. Electrophysiologic and electroanatomic changes in the human atrium associated with age. $J$ Am Coll Cardiol 2004;44:109-16.

24. Medi C, Kalman JM, Spence SJ, et al. Atrial electrical and structural changes associated with longstanding hypertension in humans: implications for the substrate for atrial fibrillation. J Cardiovasc Electrophysiol 2011;22:1317-24.

25. Oakes RS, Badger TJ, Kholmovski EG, et al. Detection and quantification of left atrial structural remodeling with delayedenhancement magnetic resonance imaging in patients with atrial fibrillation. Circulation 2009;119:1758-67.

26. Díez J. Mechanisms of cardiac fibrosis in hypertension. J Clin Hypertens 2007;9:546-50.

27. Perticone F, Ceravolo R, Pujia A, et al. Prognostic significance of endothelial dysfunction in hypertensive patients. Circulation 2001;104:191-6.

28. Yaghi S, Moon YP, Mora-McLaughlin C, et al. Left atrial enlargement and stroke recurrence: the Northern Manhattan Stroke Study. Stroke 2015;46:1488-93. 